\title{
Inclusão dos temas de gestão nos currículos dos cursos de graduação em Odontologia no Brasil
}

Inclusion of management themes in the curricula of undergraduate courses in Dentistry in Brazil

Inclusión de temas de gestión en los planes de estudio de los cursos de pregrado en Odontología en Brasil

\author{
José Jhenikártery Maia de OLIVEIRA ${ }^{1}$ \\ Nicholas Nicolau de ALMEIDA ${ }^{2}$ \\ Micaella Fernandes FARIAS ${ }^{1}$ \\ Kauana da Silva ANDRADE ${ }^{1}$ \\ Fábio Victor Dias SILVA ${ }^{1}$ \\ Marcos Aurélio Vasconcelos LIMA JÚNIOR ${ }^{3}$
}

${ }^{1}$ Graduando (a) do Curso de Graduação em Odontologia pelo Centro Universitário de João Pessoa - UNIPÊ 58053-000 João Pessoa-PB, Brasil ${ }^{2}$ Cirurgião-Dentista formado pelo Centro Universitário de João Pessoa - UNIPÉ 58053-000 João Pessoa-PB, Brasil

${ }^{3}$ Professor Mestre do Curso de graduação em Odontologia do Centro Universitário de João Pessoa - UNIPE 58053-000 João Pessoa-PB, Brasil.

\section{Resumo}

Introdução e objetivo: Considerando a escassez de disciplinas que abordem a intrínseca relação entre a área da saúde, especificamente a odontologia, e a administração, nas grades curriculares dos cursos de graduação em Odontologia espalhados pelo país, o presente estudo teve como objetivo avaliar a inclusão dos temas da área de gestão nos currículos dos cursos de graduação em odontologia, conforme determinam as Diretrizes Curriculares Nacionais de 2002. Metodologia: Tratase de uma pesquisa descritiva, exploratória, cuja população foi constituída por todas as faculdades registradas junto ao Conselho Federal de Odontologia. A coleta dos dados foi realizada através dos sites institucionais das faculdades, por meio da análise das ementas e planos de curso de graduação em odontologia, no período de junho a setembro de 2016 . Resultados: Verificou-se a existência de 220 faculdades de odontologia no Brasil. O estado de São Paulo concentrava 21,4\% das instituições; $71,69 \%$ divulgavam suas informações curriculares no site; 36,97\% ofertavam disciplinas da área de gestão e $7,56 \%$ ofertam conteúdos de gestão através de outras disciplinas. Conclusão: Em função da inobservância de um percentual expressivo de cursos de odontologia que atendam à determinação da inclusão da formação em gestão nas matrizes dos cursos de odontologia no Brasil, pode-se concluir que esta ainda é bastante incipiente e que, possivelmente, os egressos destes cursos apresentam um significativo déficit de formação.

Descritores: Educação em Odontologia; Organização e Administração; Recursos Humanos em Odontologia.

\section{Abstract}

Considering the scarcity of disciplines that address the intrinsic relationship between the health area, specifically dentistry, and administration, in the curriculum of undergraduate courses in Dentistry throughout the country, the present study aimed to assess the inclusion of themes in the area of management in the curricula of undergraduate dentistry courses, as determined by the National Curriculum Guidelines of 2002. This is a descriptive, exploratory research, whose population was constituted by all faculties registered with the Federal Council of Dentistry. The data collection was carried out through the institutional websites of the colleges, through the analysis of the menus and plans of undergraduate courses in dentistry, from June to September 2016. There were 220 dentistry colleges in Brazil. The state of São Paulo concentrates $21.4 \%$ of the institutions; $71.69 \%$ disclose their curricular information on the website; $36.97 \%$ offer management disciplines and $7.56 \%$ offer management content through other disciplines. Due to the non-observance of a significant percentage of dentistry courses that meet the determination of the inclusion of management training in the matrices of dentistry courses in Brazil, it can be concluded that this is still quite incipient and that, possibly, the graduates of these courses have a significant training deficit.

Descriptors: Education, Dental; Organization and Administration; Dental Staff.

\section{Resumen}

Introducción y objetivo: Considerando la escasez de disciplinas que aborden la relación intrínseca entre el área de la salud, específicamente la odontología y la administración, en los planes de estudio de las carreras de grado en Odontología en todo el país, el presente estudio tuvo como objetivo evaluar la inclusión temas de gestión en los planes de estudio de los cursos de licenciatura en odontología, según lo determinado por los Lineamientos Curriculares Nacionales de 2002. Metodología: Se trata de una investigación descriptiva, exploratoria, cuya población estuvo conformada por todos los colegios registrados en el Consejo Federal de Odontología. La recolección de datos se realizó a través de los sitios web institucionales de las facultades, mediante el análisis de los menús y planes de la carrera de grado en Odontología, de junio a septiembre de 2016. Resultados: Se contabilizaron 220 facultades de odontología en la Brasil. El estado de São Paulo concentró el 21,4\% de las instituciones; El $71,69 \%$ divulgó su información curricular en el sitio web; El 36,97\% ofreció cursos en el área de gestión y el 7,56\% ofrece contenidos de gestión a través de otras disciplinas. Conclusión: Debido a la no observancia de un porcentaje significativo de cursos de odontología que cumplen con la determinación de la inclusión de la formación gerencial en las matrices de los cursos de odontología en Brasil, se puede concluir que esta aún es bastante incipiente y que, posiblemente, los egresados estos cursos tienen un importante déficit de formación.

Descriptores: Educación en Odontología; Organización y Administración; Personal de Odontología.

\section{INTRODUÇÃO}

A administração na área da saúde está saindo da predominância de sua atuação na dimensão pública, abrindo espaços cada vez mais significativos na esfera privada. Considerando esse novo cenário considera-se necessário melhorar os processos de gestão no que diz respeito a sua eficiência e qualidade, visto que a competitividade passa a ser um fator relevante na sobrevivência dessas organizações ${ }^{1}$.
Nesse contexto, profissionais graduados em cursos da área da saúde, estão sujeitos a serem gestores de suas próprias organizações, pois clinicar e gerir são práticas interligadas e que se complementam ${ }^{2}$. O mercado de trabalho dos profissionais da área de odontologia é muito disputado. Dessa forma, a dimensão da competitividade passa se constituir em um fator a ser considerado. No caso da área em questão, a competitividade se articula em duas dimensões: a qualidade da atuação profissional 
relacionada ao exercício da odontologia e ainda todos os processos que envolvem a dimensão da gestão, ou seja, desde a infraestrutura, inovação, atendimento ao cliente (pré e pósvenda), preços, enfim, questões diretamente relacionadas à estrutura administrativa de um negócio ${ }^{1}$.

O problema na saúde entre formação e gestão de organizações é bem claro, mas o setor da saúde pública parece que já está despertando para o problema há mais tempo. Muitas iniciativas do setor propiciaram 0 desenvolvimento de um pensamento crítico e estimularam o fortalecimento do movimento por mudanças no processo de formação ${ }^{3}$.

Planejar a abertura de consultórios ou clínicas odontológicas é uma necessidade para os que optarem por ser profissional liberal/autônomo, dono de sua própria organização, onde a cultura empreendedora não foi formada e o sucesso da carreira depende mais do conhecimento técnico profissional, dificultando assim, para os jovens recém-formados elaborar ideias inovadoras e criativas capazes de gerar oportunidades para sua vida profissional. Oportunidade está relacionada com a criação de valor, é um conceito, ou ideia, transformado em serviço tangível, que resultará em lucro, mas as oportunidades não são iguais para todos e nem todos exploram as oportunidades ${ }^{4}$.

De 1991 a 2004 ocorreram várias mudanças na grade curricular dos cursos da área de saúde no Brasil, incluindo na graduação de odontologia. As Diretrizes Curriculares Nacionais sinalizam para uma mudança paradigmática na formação de profissional crítico, capaz de trabalhar em equipe e destacar a realidade social. Estas também definem seu objeto e propõem como perfil do profissional a ser formado: "profissional generalista, com sólida formação técnico-científica, humanística e ética, orientada para a promoção de saúde, com ênfase na prevenção de doenças bucais prevalentes" 5 .

Muitos profissionais recém-formados em odontologia encontram um início de carreira árduo e dentre as dificuldades encontradas, os problemas administrativos enfrentados pelos mesmos são muito comuns ${ }^{2}$. Tendo em vista que a formação profissional de um cirurgiãodentista é mais enfocada na técnica, o profissional geralmente sabe lidar com os problemas de saúde bucal do paciente, porém tende a ter dificuldades para administrar sua carreira e oferta serviços. As matérias no currículo mínimo têm como objetivo a formação básica, profissionalizante e social, não contendo entre elas disciplinas de ordem administrativa, salvo as do serviço de saúde coletiva. Diante disso, a falta de disciplinas eficazes na área de gestão nas grades curriculares dos cursos de odontologia é um dos fatores precursores para tais dificuldades.

Este trabalho teve como objetivo avaliar a inclusão dos temas da área de gestão nos currículos dos cursos de graduação em odontologia, conforme determinam as Diretrizes Curriculares Nacionais de $2002^{6}$, verificando o número de cursos de graduação em odontologia ofertados no Brasil; o cumprimento determinado da inclusão da formação em gestão nas matrizes dos cursos de Odontologia no Brasil; e a evolução da inclusão desses conteúdos nos cursos.

MATERIAL E MÉTODO

Trata-se de uma pesquisa descritiva e exploratória, tendo uma população constituída por todas as faculdades registradas junto ao Conselho Federal de Odontologia - CFO, conforme disponibilizado no site institucional (www.cfo.org.br).

A coleta dos dados foi realizada através dos sites institucionais das faculdades, por meio da análise das ementas e planos de curso de graduação em odontologia, no período de junho a setembro de 2016.

Os endereços dos sites foram coletados na página eletrônica do CFO por unidade da federação, assim como a quantidade de vagas disponíveis ao ano e o tipo da instituição quanto à pública federal, pública estadual, pública municipal ou particular.

Das entidades que possuíam site ativo, foram retirados dados como: unidade da federação a qual fazia parte, duração mínima do curso por semestre, disponibilidade de disciplinas específicas da área de gestão, disciplinas não específicas que apresentavam o conteúdo de gestão, obrigatoriedade destas e semestre no qual eram ministradas.

Após obtenção dos dados por entidade, foi possível tabular os mesmos através de uma planilha com o apoio das ferramentas do Microsoft Office Excel.

RESULTADOS

Por meio da análise de informações obtidas do Conselho Federal de Odontologia no ano de 2015, constatou-se a existência de 220 faculdades em funcionamento. O estado que apresentou maior número de instituições foi São Paulo, totalizando 47 (21,4\%). Os estados do Acre e Roraima foram os que apresentaram o menor número, com apenas uma unidade cada. A região que mais concentra faculdades de 
odontologia no Brasil é a região sudeste $(43,64 \%)$, e a região com menor número é a centro-oeste (8,64\%).

Em todas as regiões as IESs particulares figuraram como maioria (75\%), principalmente na região sudeste, que apresentou $78,15 \%$ das suas instituições pertencentes ao setor privado de ensino. A região nordeste, por sua vez, elencou a menor proporção de instituições privadas $(62,79 \%)$.

Em segundo lugar, com um percentual bem mais baixo que o anterior, estão as faculdades públicas federais, ocupando 12,73\% da totalidade. Neste caso, a região que melhor representou 0 valor percentual foi a nordeste, com $23,26 \%$ de suas instituições sob administração do poder público federal.

As instituições de menor ocorrência foram as públicas municipais, com 3,34\% em todo o país, sendo a maior concentração destas observadas no Sudeste com 5,21\%, mais precisamente no estado de São Paulo.

Das instituições, $\quad 9,09 \%$ não disponibilizavam site ativo contendo as informações acadêmicas necessárias para a coleta dos dados.

As regiões nordeste e sudeste foram as que apresentaram os maiores percentuais de instituições com sites ativos (93,02\% e 92,71\%). A maior proporção de faculdades sem endereço eletrônico foi observada entre as faculdades da região norte $(17,18 \%)$.

Com relação ao tipo das faculdades, $92,73 \%$ das universidades privadas e $96,43 \%$ das federais ofertaram os sites institucionais no momento da coleta dos dados. Já nas públicas estaduais esse percentual foi de $84,21 \%$. As públicas municipais, em sua totalidade, apresentavam os endereços eletrônicos ativos. As disciplinas específicas da área por gestão foram representadas na Tabela 1.

Tabela 1. Disciplinas específicas da área de gestão por regiõe
\begin{tabular}{c|c|c|c|c|c|c}
\hline \multicolumn{2}{c}{ Disciplinas específicas da área de gestão por regiões } \\
\hline \multirow{2}{*}{ Regiões } & \multicolumn{2}{|c|}{ Sim } & \multicolumn{2}{c|}{ Não } & \multicolumn{2}{c}{ Total } \\
\cline { 2 - 7 } & $\mathbf{N}$ & $\mathbf{\%}$ & $\mathbf{n}$ & $\mathbf{\%}$ & $\mathbf{N}$ & $\mathbf{\%}$ \\
\hline Norte & 6 & 50 & 6 & 50 & 12 & 100 \\
Centro-oeste & 11 & 73 & 4 & 27 & 15 & 100 \\
Nordeste & 7 & 47 & 8 & 53 & 15 & 100 \\
Sudeste & 20 & 35 & 37 & 65 & 57 & 100 \\
Sul & 9 & 33 & 18 & 67 & 27 & 100 \\
\hline Total & $\mathbf{5 3}$ & $\mathbf{4 2}$ & $\mathbf{7 3}$ & $\mathbf{5 8}$ & $\mathbf{1 2 6}$ & $\mathbf{1 0 0}$ \\
\hline
\end{tabular}

Das faculdades que possuíam sites ativos, $42 \%$ continham disciplinas específicas da área de gestão em sua grade curricular. Nas instituições da região centro-oeste observou-se que a maioria ofertava tais disciplinas (73\%), enquanto que no Sul foi verificada a menor proporção (33\%). Na região sudeste encontrouse o maior número absoluto de instituições que continham em seu elenco de disciplinas, as temáticas da área de gestão (20 - 35\%).

O Gráfico 1 apresenta a evolução da inclusão dos temas de gestão nos cursos de Odontologia no Brasil. Ressalta-se que a análise tomou como base apenas as disciplinas específicas da área de gestão.

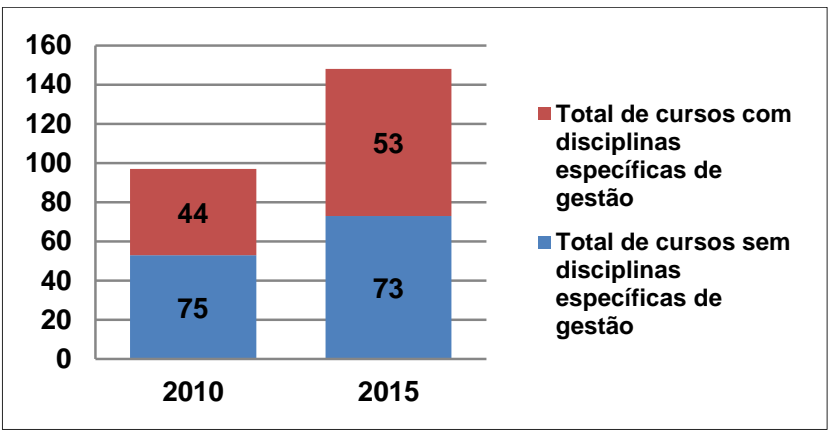

Gráfico 1: Evolução da inclusão de disciplinas específicas da área de gestão nas matrizes dos cursos de graduação em Odontologia no Brasil (Fonte: Dados da Pesquisa, 2016).

Dos 148 cursos de graduação avaliados, apenas 53 contemplam a temática da gestão em suas matrizes, atendendo ao preconizado pelas DCNs de 2002; 13 anos depois. O Gráfico 2 apresenta a evolução da inclusão de disciplinas específicas de gestão nas matrizes dos cursos de odontologia, por regiões do Brasil.

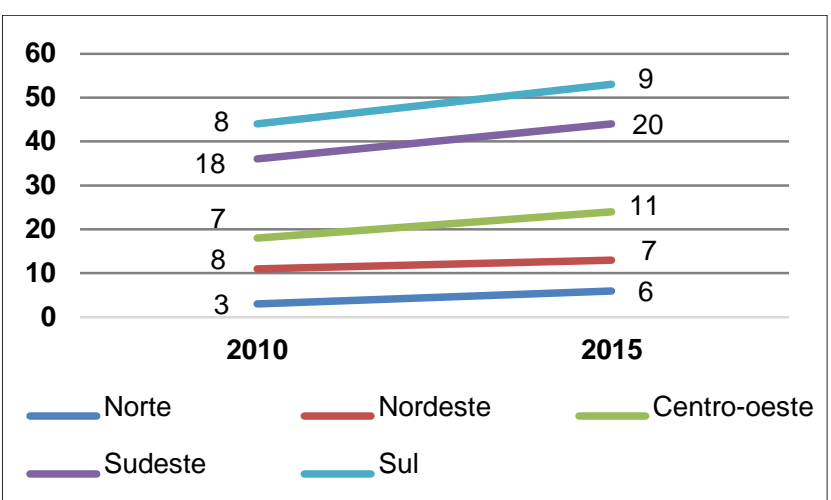

Gráfico 2: Evolução da inclusão de disciplinas específicas da área de gestão nas matrizes dos cursos de graduação em Odontologia por região.

Percebe-se que, exceto no Nordeste, houve melhora nesse percentual, principalmente na região centro-oeste. No Nordeste, tal involução foi influenciada pelo fechamento de cursos de graduação. A Tabela 2 descreve de forma detalhada o cenário da inclusão das disciplinas por regiões específicas.

Percebe-se que na região Norte, dobrou a quantidade de cursos com disciplinas específicas de gestão e, ainda, houve redução de cursos sem disciplinas específicas de gestão, o que mostra, de fato, um progresso no sentido a atender às demandas educacionais 
propostas pelas Diretrizes Curriculares dos Cursos de Odontologia do Brasil.

A região Nordeste, apresentou uma involução tanto no que concerne à quantidade de cursos com disciplinas específicas de gestão como àqueles sem disciplinas específicas de gestão. Esse cenário pode ser explicado pelo fechamento de instituições de ensino de odontologia na região. Diferente da região Centro-oeste, que apresentou a maior evolução no que diz respeito à questão elencada no título da tabela. Já na região Sul, houve aumento tanto de cursos com disciplinas específicas de gestão quanto sem disciplinas específicas de gestão.

Após essa análise, visualiza-se que as grades curriculares dos cursos de odontologia no Brasil ainda deixam a desejar no que se refere à inclusão de disciplinas de gestão, o que compromete à qualidade dos profissionais formados por essas instituições de ensino.

Tabela 2. Evolução da inclusão de disciplinas específicas da área de gestão nas matrizes dos cursos de graduação em Odontologia por região

\begin{tabular}{c|c|l|c|c}
\hline Região & $\begin{array}{c}\text { Sem } \\
\text { Disciplina } \\
2010 \\
\mathrm{n}\end{array}$ & $\begin{array}{c}\text { Com } \\
\text { Disciplina } \\
2010 \\
\mathrm{n}\end{array}$ & $\begin{array}{c}\text { Sem } \\
\text { Disciplin1 } \\
2015 \\
\mathrm{n}\end{array}$ & $\begin{array}{c}\text { Com } \\
\text { Disciplina } \\
2015 \\
\mathrm{n}\end{array}$ \\
\hline Norte & 7 & 3 & 6 & 6 \\
\hline Nordeste & 9 & 8 & 8 & 7 \\
\hline Centro-oeste & 5 & 7 & 4 & 11 \\
\hline Sudeste & 37 & 18 & 37 & 20 \\
\hline Sul & 17 & 8 & 18 & 9 \\
\hline Total & $\mathbf{7 5}$ & $\mathbf{4 4}$ & $\mathbf{7 3}$ & $\mathbf{5 3}$ \\
\hline
\end{tabular}

DISCUSSÃO

O estado de São Paulo foi o que apresentou maior número de faculdades no Brasil, provavelmente devido ao seu crescimento populacional e principalmente por ser um polo de desenvolvimento constante, o que contribuiu para que o sudeste seja a região onde mais se concentram faculdades no país.

As faculdades particulares elencaram o maior número em todas as regiões. As regiões apresentaram números de vagas anuais proporcionais ao número de faculdades, sendo a região sudeste a que mais oferta vagas e 0 centro-oeste menos, porém, ao analisar o número de vagas por habitante, o resultado surpreende. É tendencioso imaginar que a região mais carente em ensino superior seja a norte, que antigamente era considerada uma região remota, mas, ao avaliar os cruzamentos vagas por habitantes, é possível constatar que a região norte é a que mais disponibiliza vagas e a nordeste menos, onde é possível sugerir maiores investimentos em faculdades na região. A duração dos cursos é mais estendida nas faculdades de gestão pública, enquanto nas particulares conclui-se em menor tempo, possivelmente para atrair mais estudantes. Já que os investimentos acadêmicos são bastante elevados, reduzindo a duração do curso tornase mais acessível.

Das faculdades que possuíam sites ativos e currículos contidos nestes, foi possível observar que a maioria das instituições de ensino superior corrobora com o que os autores afirmam $^{2,7-9}$. Na maioria das faculdades existe omissão sobre assuntos de gestão e praticamente o curso de odontologia é dedicado à formação técnica. Confirmando o que diz Peres et al. ${ }^{8}$, realmente os modelos curriculares da maioria das faculdades de odontologia brasileiras não encontram respaldo formativo no que diz respeito ao conhecimento de gestão, dificultando assim a atuação do cirurgiãodentista como gestor de saúde.

O número de cirurgiões-dentistas aumentou proporcionalmente a proliferação de faculdades, tornando o mercado cada vez mais competitivo $^{10}$. Como sugerem Oliveira e Vilar ${ }^{11}$ para diferenciar-se dos demais, e atingir melhor o mercado, o profissional deverá procurar respaldo em conhecimentos que vão além dos oferecidos pelas instituições de ensino.

Quando analisando o que preconiza as Diretrizes Curriculares, é possível ver claramente que as faculdades que não ofertam disciplinas com conteúdos de gestão descumprem com 0 direcionamento das Diretrizes, pois, de acordo com as mesmas, o trabalho dos profissionais de saúde, dos cursos de graduação em odontologia deve estar fundamentado na capacidade de tomar decisões, visando o uso apropriado, eficácia e custo-efetividade da força de trabalho, de medicamentos, de equipamentos, de procedimentos e de práticas, ou seja, deve estar fundamentado na gestão em saúde ${ }^{5}$.

Como 0 surgimento das Diretrizes Curriculares aboliu o currículo mínimo, fica difícil para as faculdades saberem exatamente as disciplinas que precisam incluir em seu acervo, mas cabe acrescentar que a inclusão de conteúdos de gestão nas faculdades de odontologia não é utopia, é fundamentada e principalmente amparada pela idealização das Diretrizes Curriculares para a formação do cirurgião-dentista como profissional apto a fazer o gerenciamento e administração tanto da força de trabalho, dos recursos físicos e materiais e de informação, da mesma forma que deverão estar aptos a serem gestores, empregadores ou líderes de equipe de saúde ${ }^{5}$. 
Os resultados deste estudo ajudarão a orientar gestores e estudantes de IESs sobre a importância e necessidade da abordagem dos temas de gestão nos cursos de bacharelado em Odontologia, garantindo uma melhor preparação para o mercado de trabalho. Para tanto, pelo fato do estudo apresentar desenho transversal, sugere-se que novos estudos sejam realizados abordando dados retrospectivos e prospectivos da tema em questão.

CONCLUSÃO

No Brasil, existem 220 faculdades registradas junto ao Conselho Federal de Odontologia, no período da devida pesquisa. A maioria não dispõe de conteúdos de gestão inseridos em sua grade curricular, sendo que nas que possuem, estes são ofertados prioritariamente em disciplinas específicas da área de gestão, com caráter obrigatório e entre - $7^{\circ}$ e $8^{\circ}$ períodos; e houve evolução na inclusão dos temas de gestão nos cursos de Odontologia ao se fazer um comparativo entre os anos de 2010 e 2015. Em função da inobservância de um percentual significativo de cursos de odontologia que atendam à determinação da inclusão da formação em gestão nas matrizes dos cursos de odontologia no Brasil, pode-se concluir que esta ainda é bastante incipiente e que, possivelmente, os egressos destes cursos apresentam um significativo déficit de formação. Sendo assim, pode-se afirmar que, neste aspecto, as Diretrizes Curriculares dos Cursos de Odontologia no Brasil não estão sendo atendidas.

\section{REFERÊNCIAS}

1. Ceccon MF. A odontologia em prova. Rev Assoc Paul Cir Dent. 2000; 54(5):353-63.

2. Limeira FIR, Rebouças PRM, Rocha EALSS, Catão MHCV. O ensino de gestão nos cursos de graduação em Odontologia no Brasil. Rev ABENO, 2018;18(1):161-69.

3. Ceccim RB, Feuerwerker LCM. O quadrilátero da formação para a área da saúde: ensino, gestão, atenção e controle social. Rev saúde coletiva, 2004;14(1):41-65.

4. Hamel G, Prahalad CK. Competindo pelo futuro. Rev adm empres. 1995;35(5):83-4.

5. Brasil. Ministério da Educação; Ministério da Saúde (2006). Trajetória dos cursos de graduação na saúde 1991 - 2004. Brasília: INEP/MEC. Disponível em: http://portal.inep.gov.br/informacao-dapublicacao//asset_publisher/6JYIsGMAMkW1/document/ id/488631. Acesso em: 12 de maio de 2020.
6. Brasil. Ministério da Educação. Conselho Nacional de Educação (2002). Câmara de Educação Superior. Resolução CNE/CES, 19 de fevereiro de 2002. Institui as Diretrizes Curriculares Nacionais do Curso de Graduação em Odontologia. Brasília. Disponível em: http://portal.mec.gov.br/cne/arquivos/pdf/CES 032002.pdf. Acesso: 15 de maio de 2020.

7. Garbin CAS, Saliba NA, Moimaz SAS, Santos KT. O papel das universidades na formação de profissionais na área de saúde. Rev ABENO. 2006;6(1):6-10.

8. Peres AS, Bastos JRM, Lauris JRP, Silva RPR, Silva HM, César Lopes Junior C. Responsabilidade administrativa do gestor de saúde. Rev ABENO, 2006;6(1):42-8.

9. Kassis E. Porque alguns fazem sucesso em Odontologia. São Paulo: Tota; 2007.

10. Silva RHA, Maia Júnior AF, Pereira AFF, Capelozza ALA, Dossi AP, Silva AA et al. Orientação profissional para o cirurgiãodentista: ética e legislação. São Paulo: Santos; 2011.

11. Oliveira IF, Vilar RV. Mercado de trabalho em constante transformação: conheça as novas discussões e reformule suas ideias. Prêmio Sinog de Odontologia - Trabalhos premiados 2005 a 2009. São Paulo; 2010.

\section{CONFLITO DE INTERESSES}

Os autores declaram não haver conflitos de interesse

AUTOR PARA CORRESPONDÊNCIA

\section{José Jhenikártery Maia de Oliveira}

Centro Universitário de João Pessoa (UNIPÊ)

Rodovia BR-230, km 22, S/N - Água Fria

58053-000 João Pessoa - PB, Brasil

Telefone: +55 (83) 99696-0759

E-mail: jhenikarterymaia@gmail.com
Submetido em 18/08/2020 Aceito em 06/10/2020 